\title{
Alloantigen-based AIDS vaccine: revisiting a "rightfully" discarded promising strategy
} Gene M. Shearer ${ }^{1 *}$ and Adriano Boasso ${ }^{2}$

\begin{abstract}
Addresses: ${ }^{1}$ Experimental Immunology Branch, Center for Cancer Research, National Institutes of Health, Building 10, Room 5A31, 10 Center Drive, Bethesda, MD 20892, USA; ${ }^{2}$ Imperial College London, Immunology Section, Chelsea and Westminster Hospital, 369 Fulham Road, London SW10 9NH, UK

* Corresponding author: Gene M. Shearer (shearerg@dc10a.nci.nih.gov)

FI000 Medicine Reports 20II, 3:12 (doi:10.3410/M3-12)

This is an open-access article distributed under the terms of the Creative Commons Attribution-Non Commercial License (http://creativecommons.org/licenses/by-nc/3.0/legalcode), which permits unrestricted use, distribution, and reproduction in any medium, provided the original work is properly cited. You may not use this work for commercial purposes.

The electronic version of this article is the complete one and can be found at: http://fl000.com/reports/m/3/I2

Abstract

This report revisits the accidental discovery that protection against simian immunodeficiency virus (SIV) infection in the early successful experimental AIDS vaccine studies in Rhesus macaques was due to antibodies directed against human leukocyte antigens (HLAs). The inactivated virus vaccine approach was discarded because protection was due to the host's immune reaction against the HLA acquired by SIV from the human cell lines in which it was grown, rather than against antigenic determinants of SIV itself. Subsequent studies have revealed that immune recognition of HLA on uninfected leukocytes also induces other factors that inhibit infection by both SIV and the human immunodeficiency virus. Pro and con aspects of immunization against HLA as a potential AIDS vaccine strategy are discussed.
\end{abstract}

When a negative control turns out to be positive It was 1991-less than ten years after the cause of AIDS had been identified-and researchers already thought they might have a successful vaccine. Evidence from several laboratories suggested that it was possible to develop a vaccine against HIV by inoculating individuals with a crippled version of the virus that could not replicate, a strategy similar to that used to produce measles, mumps, and polio vaccines. In their tests, researchers used a virus similar to HIV, called simian immunodeficiency virus (SIV), which infects Rhesus macaque monkeys. Over time the monkeys would develop AIDS-like symptoms, much like humans. Researchers inactivated SIV, injected it into the monkeys as a vaccine, and tested whether the animals were protected against live SIV infection. Indeed, several monkeys were protected, and AIDS researchers were encouraged that an effective human AIDS vaccine would soon follow.

However, in October 1991, a brief article was published that sent AIDS vaccine research into a tailspin [1]. Like others [2,3], E. James Stott's laboratory had immunized macaques with inactivated SIV, which protected them against subsequent infection with live virus. In contrast to earlier reports, the Stott laboratory included a negative control that was missing from the earlier studies. Thus, a second group of monkeys was immunized with the human host cells that had been used to grow the inactivated SIV, but which had not been infected with SIV and, therefore, contained no trace of the virus [1]. The purpose of this negative control was to ensure that the immune reaction, which had successfully protected the monkeys, was specific for SIV antigens, and was not induced by something other than SIV. Surprisingly, the "negative control" produced protective immunity against SIV infection. Equally surprising was the fact that protection in the vaccine group was not associated with antibodies that recognized SIV antigens.

Stott's discovery prompted additional experiments to elucidate the underlying mechanisms of protection. First, HLA class I and II molecules were shown to be expressed on the surface of HIV and SIV-enveloped viruses such as HIV and SIV are coated with a lipid membrane that is stolen from the host cell as the virus exits, and this coat includes host membrane proteins including HLA. 
Interestingly, the amounts of HLA on the viruses' envelope exceeded those of the main viral antigen gp120 [4]. Second, immunization of macaques with purified HLA class II protected the animals from infection with SIV that bore the same HLA [2] but not against SIV grown in cells that expressed different HLA. Although some inactivated virus vaccine studies also reported SIV-specific antibody responses [1], the protection achieved in these studies was not due to a classic vaccine effect, intended to generate immunologic memory against specific viral antigens. Rather, a nonSIV-specific experimental "artifact", namely the immune reaction against xenogeneic HLA, was shown to be the cause of the protection.

Despite the fact that more than 200 macaques in several independent studies were protected from infection [3], presumably by anti-HLA antibodies, the whole inactivated virus AIDS vaccine approach was largely discarded in favor of pursuing vaccines that would generate HIV/ SIV-specific adaptive humoral and cellular immunity. This was a sensible and rational decision at the time, taken by following the rigorous scientific process of rejecting the initial hypothesis based on the experimental results and controls. If now, almost 30 years after HIV was isolated, an effective virus-specific vaccine against HIV were available, there would be no reason to look back at those experiments and revisit their outcome. However, the limited success of some promising-looking HIV vaccine trials in recent years prompted us to reflect and reconsider retrospectively some of the earlier studies on HIV/SIV vaccines. Can we learn anything from the failed negative control of the Stott laboratory's experiment? Would we still consider the outcome of that study to be an artifact, if instead of searching for anti-SIV responses, we could reformulate the hypothesis and postulate that anti-HLA reactions can protect against SIV/ HIV infections? As early on as 1993, a letter in Science suggested that the protection induced by foreign HLA activation should be pursued as a possible AIDS vaccine strategy [5].

\section{Other HLA-activated anti-SIV/HIV mechanisms}

Might immunologic factors other than anti-HLA antibodies have been activated by foreign HLA recognition? Such mechanisms may have been overlooked or not detected in the earlier SIV/macaque studies, but could have contributed to the observed protection against infection. More recent independent studies discovered multiple protective roles for xenoantigen- and alloantigen-activated immunity. These components included anti-CCR5 (C-C chemokine receptor type 5) antibodies [6], and several innate antiviral factors in both the SIV/macaque and HIV/human experiments. These factors include the CD8
T cell-derived anti-HIV factor (CD8-SF) [7] and the $\beta$-chemokines RANTES (Regulated upon Activation, Normal T-cell Expressed, and Secreted), MIP-1 $\alpha$ (macrophage inflammatory protein- $1 \alpha$ ), and MIP-1 $\beta$ [8], which block the CCR5 coreceptor, essential for virus entry into target leukocytes. These results raise the possibility that anti-SIV factors and anti-CCR5 antibodies also contributed to protection against infection in the early vaccine studies. Intentional in vivo alloimmunization of recurrent spontaneously-aborting women against their partners' peripheral blood leukocytes induced: (a) increased levels of RANTES, MIP-1 $\alpha$, MIP-1 $\beta$, and CD8-SF; (b) decreased CCR5 and CXCR4 (C-X-C chemokine receptor type 4) coreceptor expression; and (c) increased resistance to in vitro HIV infection [8], a picture that resembled the results obtained by xenogeneic immunization in the SIV/macaque model.

HLA alloantigen stimulation of human peripheral blood leukocytes also induced the soluble factor EDN (eosinophil-derived neurotoxin) in monocytes [9] and the cytodine deaminase APOBEC3G (A3G) in CD4 ${ }^{+} \mathrm{T}$ cells [10]. Both EDN and A3G interfere with HIV replication in the crucial window of opportunity after virus entry and before reverse transcription. This effect can also been seen in vivo, in alloimmunization of recurrent spontaneously-aborting women, for example, which resulted in increased A3G that was associated with reduced CD4 ${ }^{+}$ T-cell susceptibility to HIV infection in vitro [10].

A study of vertical perinatal (i.e., from mother to baby during delivery) transmission of HIV in Kenya indicated that babies with HLA class I similar to that of their mother were more likely to contract HIV [11]. Similar results were obtained in a mother/neonate study in the United States. Several cohorts of individuals have been identified that are repeatedly exposed to HIV, but appear not to have become infected [12]. One cohort of HIVexposed seronegative patients was identified in couples in which only one partner is HIV-infected, and who do not use safe sexual practices. These HIV-exposed seronegative individuals produced anti-CCR5 serum antibodies [13], and their peripheral blood leukocytes exhibited elevated levels of A3G [14]. Thus, HLA alloantigen recognition might contribute to protecting against both vertical and horizontal HIV transmission.

None of these antiviral mechanisms may be, on its own, sufficient to provide effective protection from SIV/HIV infection. For example, immunization of macaques against CCR5 provided partial protection from SIV challenge, but did not achieve the same levels of protection that had been observed in studies using inactivated SIV vaccine [15]. However, when several mechanisms such as the ones described above are activated simultaneously, 
Table I. Advantages and disadvantages of alloantigen-based AIDS vaccines

\begin{tabular}{ll}
\hline Advantages & Disadvantages \\
\hline $\begin{array}{l}\text { Induces potent anti-HLA antibody memory immunity } \\
\text { Inactivated xenogeneic SIV induces anti-SIV antibodies as well as other }\end{array}$ & $\begin{array}{l}\text { Could exclude vaccine recipients from receiving tissue transplants } \\
\text { Might induce autoimmune or other detrimental immune conditions }\end{array}$ \\
$\begin{array}{l}\text { mechanisms } \\
\text { Inactivated xenogeneic SIV and xenogeneic cells already shown to protect }\end{array}$ & $\begin{array}{l}\text { Could induce high frequency of HLA-specific CD4 }{ }^{+} \text {virus target cells } \\
\text { against SIV infection ( }>200 \text { animals) }\end{array}$ \\
$\begin{array}{l}\text { Alloimmunization of recurrent spontaneously-aborting women }(>2,500) \\
\text { did not show significant adverse side effects, but did show reduced in }\end{array}$ & $\begin{array}{l}\text { Innate anti-HIV factors might not exhibit immunological memory } \\
\text { vitro HIV infection }\end{array}$ \\
$\begin{array}{l}\text { Immunologically indifferent to viral mutation } \\
\text { Induces several different innate antiviral factors, including CD8-SF, RANTES, }\end{array}$ & $\begin{array}{l}\text { Need to be sterilizing: if infection occurs, donor HLA is rapidly replaced } \\
\text { by host HLA }\end{array}$ \\
Induces CCR5 antibodies and reduces HIV coreceptor expression & Immunity is not virus specific \\
\hline
\end{tabular}

CCR5, C-C chemokine receptor type 5; CD8-SF, cluster of differentiation 8-supressor factor; EDN, eosinophil-derived neurotoxin; HLA, human leukocyte antigen; MIP-I $\alpha$, macrophage inflammatory protein-I $\alpha$; RANTES, Regulated upon Activation, Normal T-cell Expressed, and Secreted; SIV, simian immunodeficiency virus.

they may synergize in conferring a protective effect. Should a vaccine strategy that generates the above arsenal of multiple innate and antibody-mediated protective mechanisms against SIV/HIV be seriously considered as an AIDS vaccine? This question can be now raised retrospectively, since the induction of the anti-HIV/SIV factors by xenoantigen or alloantigen recognition was reported only after the inactivated virus vaccine strategy had been dropped.

\section{The virus-specific AIDS vaccine approach}

In March 2008, the Division of AIDS (DAIDS), National Institute of Allergy and Infectious Diseases (NIAID) held a summit meeting in Bethesda, Maryland, to discuss the repeated failures that spanned a 20 -year period of attempts to develop an effective HIV antigen-specific prophylactic AIDS vaccine, and to consider plans for the future. Our subsequent commentary on the summit suggested that innovative nontraditional approaches not bound to the paradigm of prime-boost stimulation of adaptive immunity should be considered in order to overcome some of the characteristic features that allow HIV to escape immune control [16].

Among the problems that undermine the effectiveness of virus-specific AIDS vaccines is the fact that HIV bypasses virus-specific immunity by mutating away from the immunogenic viral epitopes for which a vaccine was designed. Another important hurdle for adaptive immunity is the rapid rate at which HIV and SIV infect mucosal sites. Thus, by infecting macaques intravaginally with SIV, Ashley Haase's laboratory estimated that endocervical infection occurs within a few hours of viral challenge, resulting in rapid migration of infection-susceptible $\mathrm{CD}^{+} \mathrm{T}$ cells to the site of viral exposure [17]. These findings suggest that adaptive immune responses induced by AIDS vaccines may be "too late, too little."
Therefore, upon exposure to infectious HIV/SIV, protective mechanisms should already be active at the site of viral challenge during this brief window of opportunity.

\section{Advantages and disadvantages of alloantigen-based AIDS vaccines}

The potential protective effect of an alloantigen-based AIDS vaccine must be considered alongside the disadvantages that this approach may have. Predicted advantages and disadvantages of alloantigen-based AIDS vaccines (inactivated allogeneic HIV and/or uninfected allogeneic leukocytes) are listed in Table 1, and are summarized in the text below.

HLA is one of the most immunogenic molecules known. Recognition of allogeneic leukocytes uniquely leads to rapid primary HLA-specific responses, and immunization with either allogeneic leukocytes or inactivated SIV induces potent anti-HLA antibody responses. Thus, based on the high density and immunogenic potential of HLA on the HIV envelope [4], anti-HLA antibodies are likely to react against HIV more efficiently than antibodies against viral envelope antigens. Notably, immunization with purified HLA was sufficient to achieve protection from infection [2], demonstrating the potency of anti-HLA antibodies in neutralizing SIV. However, when inactivated HLA-bearing SIV was used as a vaccine by Stott and others, they observed not only anti-HLA protective antibodies, but also anti-SIV antibodies that were induced by SIV antigens expressed by the immunizing HIV particles [1,3]. Therefore, the inactivated HIV/SIV approach may meet the requirement for both antiviral and anti-HLA immunity. Noteworthy here is the fact that those experiments of HLA-associated immunity protected macaques from intravenous infection with high doses of virus [1,3]. Whether anti-HLA, anti-CCR5, and other innate antiviral factors may be 
present at mucosal level in amounts sufficient to protect from infection via sexual exposure is still unknown. It is possible that the efficacy of HLA immunization might even be higher against natural exposure to moderate (or suboptimal) doses of virus.

An attractive feature of alloantigen-based AIDS vaccines is that, because it would not rely on HIV epitopes, alloimmunization would be indifferent to viral mutation. Furthermore, the innate antiviral factors and anti-CCR5 antibodies induced by immunization with allogeneic leukocytes inhibit SIV and HIV replication at different points in the viral cycle. This may increase the pressure on HIV, which is less likely to develop mutations that would escape the multiple inhibitory mechanisms activated by alloantigen immunization [6-10]. Most importantly, if anti-HLA and anti-CCR5 antibodies generated by alloantigen-based AIDS vaccines can be induced at mucosal sites at the time of infectious challenge, they may exert protective activity during this narrow window of opportunity, bypassing the "too late or too little" problem [17].

Among the arguments against using alloantigen-based AIDS vaccines are the facts that allogeneic immunization might induce autoimmune or other detrimental immunologic conditions and exclude vaccine recipients from future allogeneic tissue transplantation. However, the probability that vaccine recipients would receive allografts in the future would be low in many HIV endemic regions of the world, and antirejection drugs could be used to prevent allograft rejection. Furthermore, allogeneic leukocytes are often transferred during blood transfusions, and more than 2,500 recurrent spontaneously-aborting women have received multiple immunizations (some more than 25 years ago) from their husbands without detected detrimental effects [18]. One counterargument to this observation is that recurrent spontaneously-aborting women were immunized only against their partners' HLA, whereas immunization against multiple HLA would be needed to induce protection from HIV infection. Nevertheless, detrimental effects associated with exposure to different HLA types are not commonly observed in individuals who have received multiple transfusions.

The fact that the HLA types on the surface of HIV with which an individual will make contact would be those of the infecting host, and therefore cannot be predicted, presents a problem for alloantigen-based AIDS vaccines. One possible solution would be to engineer a group of maximally-discordant HLA haplotypes into a selected target leukocyte cell line. This cell line could then be used as the vaccine itself, or as the source of HIV/SIV particles to be utilized as the vaccine. Ideally, such vaccines could generate anti-HLA antibodies to protect against HIV/SIV expressing several different HLA. Another pitfall of the alloantigen-based AIDS vaccine approach may be that the efficacy of protection could correlate inversely with the level of HLA matching between the vaccinated individual and the infecting virus. Thus, one would expect that, if the vaccinated individual and infecting HIV were perfectly HLA matched, there would be no protective effect provided by anti-HLA antibodies. In this rare situation, anti-HIV activity might be limited to the effect of alloantigen-based AIDS vaccine-induced anti-CCR5 coreceptor antibodies. Thus, the lack of HLA alloantigen recognition would not activate antiviral factors, and vaccine-induced anti-HLA antibodies would not recognize the matched HLA on the infecting virus as allogeneic. On the contrary, anti-HLA antibody-mediated protection and innate antiviral factors would be highest with maximal HLA discordance between the vaccinated individual and the infecting virus. Different degrees of protection might be observed between these two extremes, depending on the level of HLA matching.

Another potential problem with alloantigen-based AIDS vaccines is the relatively high frequency of HLA-specific $\mathrm{CD}^{+} \mathrm{T}$ cells, which could be activated by immunization against HLA alloantigens and may result in an increased number of potential targets for HIV infection. The problem of increased $\mathrm{CD} 4^{+} \mathrm{T}$ cell targets for infection, however, would also be shared by other vaccine strategies, including vector-based HIV-specific vaccines [19]. Whether an increase in the number of targets for infection could be counteracted by the multiple protective mechanisms that an alloantigen-based AIDS vaccine would induce is unknown. The only clue comes from early macaque studies involving xenoimmunization showing that anti-HLA antibody [1-3], and probably innate antiviral factors and anti-CCR5 antibodies, appeared to overcome any increase in HLA-specific $\mathrm{CD} 4^{+} \mathrm{T}$-cell targets, ultimately protecting the host from infection.

One major concern for the immune responses induced by alloantigen-based AIDS vaccines is whether they would induce long-lasting protection and immunologic memory. Anti-HLA antibody responses are known to be maintained for several years. HLA alloimmunization of recurrent spontaneously-aborting women indicated that reduced CCR5 coreceptor expression was maintained for at least 12 months, the period of follow-up after alloimmuniztion [8]. However, innate responses may be different, because the same study indicated that the $\beta$-chemokines were detected for only 6 months after alloimmunization [8]. Thus, the innate mechanisms that participate in the protective response may be more short-lived than adaptive immune responses. This could be advantageous because 
long-term innate responses, if achieved, might be detrimental to the host. A3G was reported in memory T cells, suggesting that this antiretroviral factor might not be so time-critical [10]. Although these innate factors might not be sufficient by themselves to protect against infection, they could contribute to protection against mucosal infection if activated in the presence of vaccine-induced pre-existing anti-HLA and anti-CCR5 antibodies. Furthermore, it is possible that once immunized against allogeneic HLA, subsequent natural exposures to HIV bearing allogeneic HLA molecules would repeatedly boost antiHLA and anti-CCR5 antibody responses, as well as rapidly reactivate the transient antiviral factors.

Another problem to be overcome is that the HLA carried by the viral envelope would remain allogeneic only as long as infection did not occur (Figure 1). If the arsenal of antiviral mechanisms induced by HLA recognition failed to prevent infection of the host target cells, newly produced HIV particles would carry the HLA of the host, which would not be recognized as foreign. Thus, the window of opportunity for an alloantigen-based AIDS vaccine to be protective would be strictly time-limited, and vaccine-induced antibodies and innate antiviral factors (the second line of defense) would optimally need to prevent infection, since they may not efficiently suppress viral replication once HIV has acquired self HLA from the host (Figure 1b). Therefore, by definition, an effective alloantigen-based AIDS vaccine would have to be sterilizing and could not permit infection to be established (Figure 1a). Again, the only clue we have as to whether this would work is the fact that more than 200 xenoantigen-immunized macaques were protected against SIV infection [3].

\section{Comparing different approaches for an alloantigen-based AIDS vaccine}

We have summarized the mechanisms by which HLA recognition may result in protection from HIV/SIV. However, it should be noted that not all of these potentially protective mechanisms were: (a) tested using both inactivated HIV/SIV and allogeneic or xenogeneic uninfected cells; (b) studied in both the SIV/macaque (xenogeneic) and HIV/human (allogeneic) models; and (c) analyzed using both in vitro and in vivo systems. These issues should be carefully investigated in comprehensive and purpose-designed in vitro and in vivo studies to properly appreciate the potential for an alloantigen-based AIDS vaccine approach. For example, it is not known whether inactivated virus would induce anti-CCR5 antibody and/or activate some or all of the innate antiviral factors induced by alloimmunization of humans in vivo. However, there are clues that it might work. Firstly, xenogeneic leukocyte immunization of macaques was reported to induce anti-HLA antibodies [1-3] and mucosal immunization of macaques with macaque allogeneic peripheral blood leukocytes (instead of xenogeneic human cell lines) activated SIV-inhibiting chemokines, increased anti-CCR5 antibody levels and was associated with decreased in vitro SIV infectivity [20]. Secondly, some of these features were seen in recurrent spontaneouslyaborting women who produced elevated levels of $\beta$-chemokines and A3G upon alloimmunization, and their $\mathrm{CD} 4^{+} \mathrm{T}$ cells were protected against in vitro infection by HIV $[8,10]$.

Would immunization using inactivated virus or purified HLA be more effective? To complete a meaningful comparative analysis of these two alloantigen-based AIDS vaccine strategies, additional in vivo macaque immunization and live mucosal SIV challenge studies may need to be performed. These studies should compare animals immunized with inactivated virus, animals immunized with purified HLA, and negative control animals for antibodies against HLA and CCR5, and for innate antiviral factors. The kinetics with which these factors are re-activated in the first hours after SIV challenge at both the mucosal and systemic levels should be monitored because of the "too late, too little" issue.

The argument can be made that xenoantigen-expressing cells may be more potent immunogens than alloantigenexpressing cells, which could have provided the protection observed in the Stott's experiment [1]. However, macaques immunized with allogeneic peripheral blood leukocytes were protected against infection by SIV grown in simian cells [2]. Additionally, a subsequent study indicated that mucosal immunization with allogeneic peripheral blood leukocytes resulted in induction of CD8-SF, MIP-1 $\alpha$, MIP-1 $\beta$ and anti-CCR5 antibody, and increased resistance to SIV infection [20]. In humans, administration of inactivated HIV-1 (Remune) as an immunotherapeutic vaccine resulted in the induction of antibody responses against HLA class I and II present in the human cell line used for virus production [21]. These findings indicate that immunization with allogeneic cells may induce protective effects similar to those obtained using xenogeneic cell lines. Nevertheless, future experiments should include testing macaque allogeneic leukocytes as a vaccine.

Although alloimmunization might induce some anti-HIV antibodies, due to the partial three-dimensional homology between HLA and HIV gp120 [5], the use of allogeneic cells instead of inactivated virus has the disadvantage of not including the generation of HIV-specific adaptive $\mathrm{T}$ cell immunity. Thus, an inactivated HIV vaccine strategy that also incorporates alloimmunization may combine 
Figure I. A successful alloantigen-based AIDS vaccine (ABAV) would need to induce sterilizing immunity

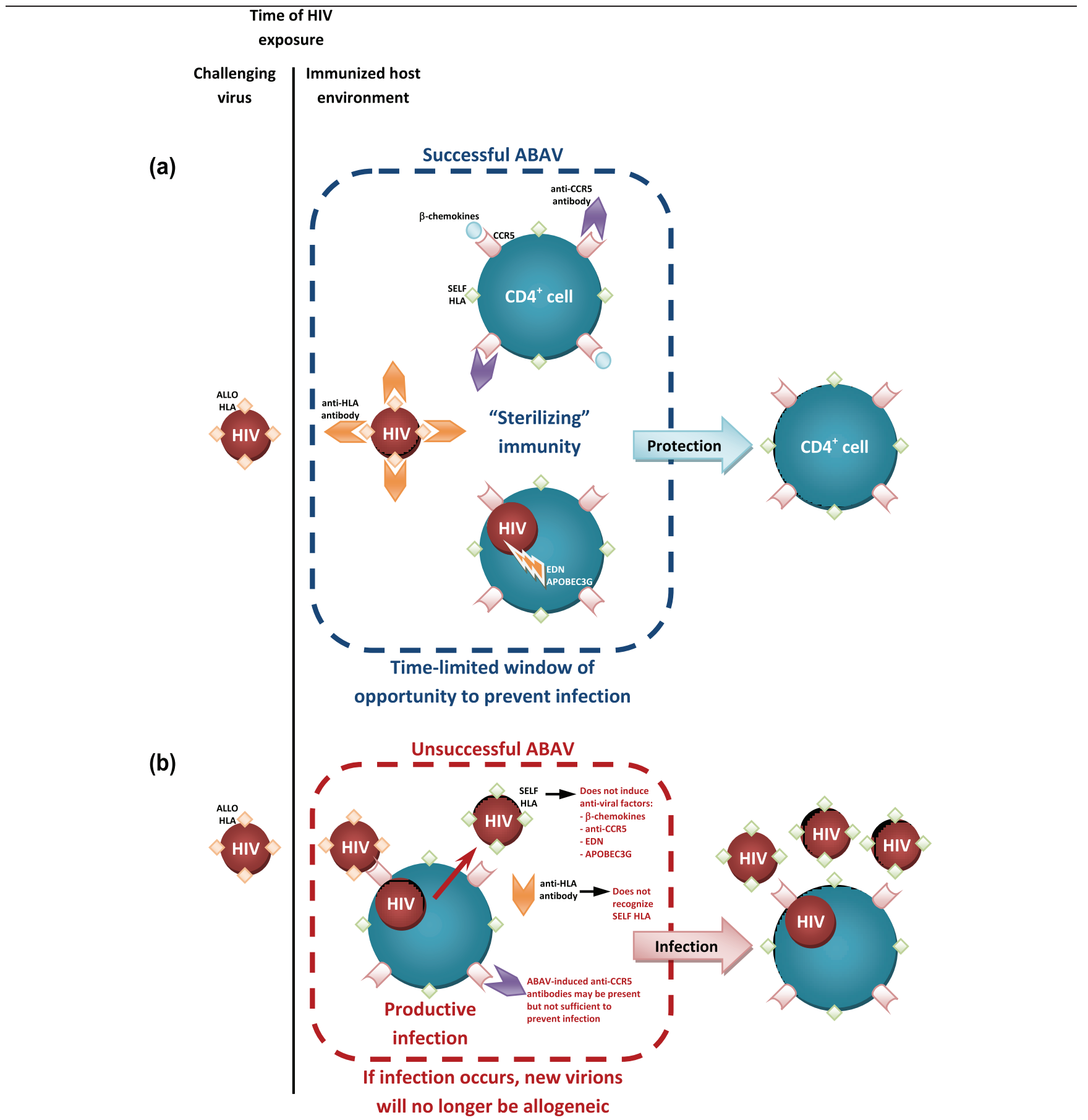

(a) Upon exposure to HIV particles carrying allogeneic human leukocyte antigen (HLA), preformed anti-HLA antibodies in the immunized host will opsonize and block challenging HIV. Anti-CCR5 (C-C chemokine receptor type 5) antibodies and $\beta$-chemokines will inhibit HIV interaction with its coreceptor and, in case HIV successfully enters target cells, intracellular restriction factors such as eosinophil-derived neurotoxin (EDN) and APOBEC3G will prevent productive infection. This allogeneic HLA-induced arsenal of antibodies and antiviral factors may efficiently prevent infection ("sterilizing" immunity) and result in full protection. (b) If alloantigen-based AIDS vaccine-induced antiviral mechanisms fail to prevent productive infection, newly produced HIV particles budding from the host's cells will carry self HLA and will not be recognized as allogeneic anymore. Anti-HLA antibodies will not react with self HLA-carrying $\mathrm{HIV}$, antiviral factors would not be produced to counteract the spread of the infection, and anti-CCR5 antibody induced by alloantigen-based AIDS vaccines might not offer sufficient protection. 
the above described benefits of an alloantigen-based AIDS vaccine with the potential for generating memory HIV-specific responses. An argument could be made that the use of allogeneic cells would avoid the risk of exposing vaccinees to incompletely inactivated HIV.

We are aware of only one international workshop on alloimmunization as an alternative AIDS vaccine strategy. That workshop was held in 1999 [18], before anticoreceptor antibodies and most of the above-noted innate anti-SIV/HIV factors were reported to result from HLA alloimmunization. The more recent and increasing body of evidence showing different alloinduced mechanisms of interference with HIV replication and infection suggest that it may be time to reassess this alternative vaccine strategy.

\section{Summary}

This report reconsiders an AIDS vaccine approach that was discarded 20 years ago because it was not virusspecific. The unorthodox and seemingly counter-scientific approach, conceptually based on a "failed" negative control, takes advantage of the facts that: (a) immunologically-potent HLA acquired from infected cells are carried on the HIV envelope; and (b) these transplantation antigens are allogeneic to the susceptible host at the time of exposure to infectious HIV. We view expression of foreign HLA as a transient Achilles heel for HIV, which creates a brief window of opportunity in which preformed, on-site anti-HLA and anti-CCR5 antibodies could initially block or limit virus entry. The additional alloantigen-based AIDS vaccine-induced arsenal of antiviral factors could provide a one-two punch to prevent productive infection.

\section{Abbreviations}

A3G, APOBEC3G; CCR5, C-C chemokine receptor type 5; CD8-SF, cluster of differentiation 8-supressor factor; EDN, eosinophil-derived neurotoxin; HLA, human leukocyte antigen; MIP-1 $\alpha$, macrophage inflammatory protein- $1 \alpha$; RANTES, Regulated upon Activation, Normal T-cell Expressed, and Secreted; SIV, simian immunodeficiency virus.

\section{Competing interests}

The authors declare that they have no competing interests.

\section{References}

I. Stott EJ: Anti-cell antibody in macaques. Nature 1991, 353:393. FI000 Factor 6 Evaluated by Gene Shearer 19 May 2010

2. Arthur LO, Bess JW Jr., Urban RG, Strominger JL, Morton WR, Mann DL, Henderson LE, Benveniste RE: Macaques immunized with HLA-DR are protected from challenge with simian immunodeficiency virus. J Virol 1995, 69:31 I7-24.

FI000 Factor 6

Evaluated by Gene Shearer 19 May 2010

3. Stott EJ, Schild GC: Strategies for AIDS vaccines. J Antimicrob Chemother 1996, 37(Suppl B): I85-98.

4. Arthur LO, Bess JW Jr., Sowder RC 2nd, Benveniste RE, Mann DL, Chermann JC, Henderson LE: Cellular proteins bound to immunodeficiency viruses: implications for pathogenesis and vaccines. Science 1992, 258:1935-8.

FI000 Factor 6

Evaluated by Gene Shearer 19 May 2010

5. Shearer GM, Clerici M, Dalgleish A: Alloimmunization as an AIDS vaccine? Science 1993, 262:161-2.

6. Lehner T, Wang Y, Doyle C, Tao L, Bergmeier LA, Mitchell E, Bogers WM, Heeney J, Kelly CG: Induction of inhibitory antibodies to the CCR5 chemokine receptor and their complementary role in preventing SIV infection in macaques. Eur J Immunol 1999, 29:2427-35.

FI000 Factor 6

Evaluated by Gene Shearer 19 May 2010

7. Wang Y, Tao L, Mitchell E, Bogers WM, Doyle C, Bravery CA, Bergmeier LA, Kelly CG, Heeney JL, Lehner T: Generation of CD8 suppressor factor and beta chemokines, induced by xenogeneic immunization, in the prevention of simian immunodeficiency virus infection in macaques. Proc Natl Acad Sci U S A 1998, 95:5223-8.

FI000 Factor 6

Evaluated by Gene Shearer 19 May 2010

8. Wang $\mathrm{Y}$, Tao L, Mitchell E, Bravery C, Berlingieri P, Armstrong P, Vaughan R, Underwood J, Lehner T: Allo-immunization elicits CD8+ T cell-derived chemokines, HIV suppressor factors and resistance to HIV infection in women. Nat Med I999, 5:1004-9.

FI000 Factor 6

Evaluated by Gene Shearer 19 May 2010

9. Rugeles MT, Trubey CM, Bedoya VI, Pinto LA, Oppenheim JJ, Rybak SM, Shearer GM: Ribonuclease is partly responsible for the HIV-I inhibitory effect activated by HLA alloantigen recognition. AIDS 2003, I 7:48|-6.

FI000 Factor 6

Evaluated by Gene Shearer 19 May 2010

10. Pido-Lopez J, Wang Y, Seidl T, Babaahmady K, Vaughan R, Lehner T: The effect of allogeneic in vitro stimulation and in vivo immunization on memory CD4(+) T-cell APOBEC3G expression and HIV-I infectivity. Eur J Immunol 2009, 39: I 956-65.

FI000 Factor 6

Evaluated by Gene Shearer 19 May 2010

II. MacDonald KS, Embree J, Njenga S, Nagelkerke NJ, Ngatia I, Mohammed Z, Barber BH, Ndinya-Achola J, Bwayo J, Plummer FA: Mother-child class I HLA concordance increases perinatal human immunodeficiency virus type I transmission. J Infect Dis |998, | 17:55|-6.

FI000 Factor 6

Evaluated by Gene Shearer 19 May 2010

12. Shearer G, Clerici M: Historical perspective on HIV-exposed seronegative individuals: has nature done the experiment for us? J Infect Dis 2010, 202(Suppl 3):S329-32.

13. Lopalco L, Barassi C, Pastori C, Longhi R, Burastero SE, Tambussi G, Mazzotta F, Lazzarin A, Clerici M. Siccardi AG: CCR5-reactive antibodies in seronegative partners of HIV-seropositive individuals down-modulate surface CCR5 in vivo and 
neutralize the infectivity of R5 strains of HIV-I In vitro. J Immunol 2000, 164:3426-33.

FI000 Factor 6

Evaluated by Gene Shearer 19 May 2010

14. Biasin M, Piacentini L, Lo Caputo S, Kanari Y, Magri G, Trabattoni D, Naddeo V, Lopalco L, Clivio A, Cesana E, Fasano F, Bergamaschi C, Mazzotta F, Miyazawa M, Clerici M: Apolipoprotein B mRNAediting enzyme, catalytic polypeptide-like 3G: a possible role in the resistance to HIV of HIV-exposed seronegative individuals. J Infect Dis 2007, 195:960-4.

FI000 Factor 6

Evaluated by Gene Shearer 19 May 2010

15. Bogers WM, Bergmeier LA, Oostermeijer $H$, ten Haaft $P$, Wang $Y$, Kelly CG, Singh M, Heeney JL, Lehner T: CCR5 targeted SIV vaccination strategy preventing or inhibiting SIV infection. Vaccine 2004, 22:2974-84.

FI000 Factor 6

Evaluated by Gene Shearer 19 May 2010

16. Boasso A, Shearer GM, Clerici M: The hunt for an HIV vaccine: time to rethink recent failures. Lancet 2008, 37I:I897-8.

17. Haase AT: Perils at mucosal front lines for HIV and SIV and their hosts. Nat Rev Immunol 2005, 5:783-92.
18. Lehner T, Shearer GM, Hackett C], Schultz A, Sharma OK: Alloimmunization as a strategy for vaccine design against HIVIAIDS. AIDS Res Hum Retroviruses 2000, 16:309-13.

19. Benlahrech A, Harris J, Meiser A, Papagatsias T, Hornig J, Hayes P, Lieber A, Athanasopoulos T, Bachy V, Csomor E, Daniels R, Fisher K, Gotch F, Seymour L, Logan K, Barbagallo R, Klavinskis L, Dickson G, Patterson S: Adenovirus vector vaccination induces expansion of memory CD4 $\mathrm{T}$ cells with a mucosal homing phenotype that are readily susceptible to HIV-I. Proc Natl Acad Sci U S A 2009, 106:19940-45.

FI000 Factor 8

Evaluated by Sheena McCormack 22 Mar 2010

20. Bergmeier LA, Babaahmady K, Wang Y, Lehner T: Mucosal alloimmunization elicits $\mathrm{T}$-cell proliferation, $\mathrm{CC}$ chemokines, CCR5 antibodies and inhibition of simian immunodeficiency virus infectivity. J Gen Virol 2005, 86:223I-8.

FI000 Factor 6

Evaluated by Gene Shearer 19 May 2010

21. Page M, Ojugo A, Imami N, Hardy G, Gotch F, Almond N: Specificity of anti-human leukocyte antigen antibody responses after immunization with Remune, an inactivated HIV-I vaccine. AIDS 2007, $21: 375-7$.

FI000 Factor 6

Evaluated by Gene Shearer 19 May 2010 